International Journal of Pure and Applied Mathematics

Volume 98 No. 3 2015, 355-364

ISSN: 1311-8080 (printed version); ISSN: 1314-3395 (on-line version)

url: http://www.ijpam.eu

doi: http://dx.doi.org/10.12732/ijpam.v98i3.8

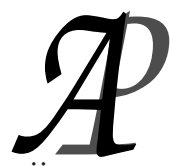

ijpam.eu

\title{
DYNAMICAL EQUIVALENCE OF QUASILINEAR EQUATIONS
}

\author{
Andrejs Reinfelds ${ }^{1,2} \S$, Dzintra Šteinberga ${ }^{2}$ \\ ${ }^{1}$ Institute of Mathematics and Computer Science of University of Latvia \\ Raiņa bulv. 29, Rīga, LV-1459, LATVIA \\ ${ }^{2}$ University of Latvia, Department of Mathematics, \\ Zellu 8, Rīga, LV-1002, LATVIA
}

\begin{abstract}
Using Green type map we can find sufficient conditions under which a quasilinear equation is dynamically equivalent to its corresponding linear equation. This result extends Grobman - Hartman theorem for equations without ordinary dichotomy.
\end{abstract}

AMS Subject Classification: 34C41, 34D09, 34G20, 37C15

Key Words: quasilinear differential equations, invertible difference equations, Grobman's-Hartman's linearization theorem, green type map

\section{Introduction}

The global version of classic D.M. Grobman - P. Hartman theorem [1], [2], [3] states that in $\mathbb{R}^{n}$ a system of differential equations

$$
\dot{x}=A x+f(x)
$$

is globally dynamically equivalent to the system of linear differential equations

Received: September 26, 2014

(C) 2015 Academic Publications, Ltd. url: www.acadpubl.eu

${ }^{\S}$ Correspondence author 


$$
\dot{x}=A x
$$

if matrix $A$ has no eigenvalues with zero real part and map $f$ is bounded and sufficiently small Lipschitzian. Let us note that the corresponding homeomorphism $H: \mathbb{R}^{n} \rightarrow \mathbb{R}^{n}$ satisfies the condition:

$$
\sup _{x}|H(x)-x| \leq c \sup _{x}|f(x)|<+\infty
$$

where the constant $c>0$ depends only on the matrix $A$. Later, K.J. Palmer [4] extended the theorem to systems of nonautonomous differential equations. He considers the case when the corresponding system of linear differential equations (3) has an exponential dichotomy [5].

Recently Grobman-Hartman-Palmer linearization theorems have been extended to systems of differential equations in $\mathbb{R}^{n}$ with generalized exponential and ordinary dichotomy in papers [6], [7], [8], [9], [10], [11].

In this work we generalize these results, even for $\mathbb{R}^{n}$, by relaxing conditions on the linear part $A$ while strengthening conditions on the nonlinear part $f$. We use Green type map and integral functional equation technique [12], [13], [14] to substantially simplify the proof. Moreover, we use completely different method to prove the dynamical equivalence. Furthermore, for more general point of view, we consider nonautonomous differential equations and nonautonomous difference equations in an arbitrary Banach space. To highlight our improvement comparing to previous results, we use an example where the linear part of the differential equation even does not possesses an ordinary dichotomy.

Using a suitable bump function and change of variables, it is possible to reduce the analysis of local equivalence of equations to investigation of the global equivalence of equations.

\section{Main Result and Proof}

Let $\mathbf{X}$ be a Banach space and let $\mathfrak{L}(\mathbf{X})$ be the Banach space of bounded linear maps. Consider the quasilinear differential equations

$$
\dot{x}=A(t) x+f_{1}(t, x)
$$

and

$$
\dot{x}=A(t) x+f_{2}(t, x)
$$

on $\mathbf{X}$, where $A: \mathbb{R} \rightarrow \mathfrak{L}(\mathbf{X})$ is integrable in the Bochner's sense. Suppose, in addition, that maps $f_{i}: \mathbb{R} \times \mathbf{X} \rightarrow \mathbf{X}, i=1,2$ are locally integrable in the 
Bochner's sense with respect to $t$ for fixed $x$, satisfy Lipschitz conditions

$$
\left|f_{i}(t, x)-f_{i}\left(t, x^{\prime}\right)\right| \leq \varepsilon(t)\left|x-x^{\prime}\right|, \quad i=1,2
$$

and

$$
\sup _{x}\left|f_{1}(t, x)-f_{2}(t, x)\right| \leq N(t)<+\infty
$$

where $N: \mathbb{R} \rightarrow \mathbb{R}_{+}$and $\varepsilon: \mathbb{R} \rightarrow \mathbb{R}_{+}$are integrable scalar functions. Let $x_{i}(\cdot, s, x): \mathbb{R} \rightarrow \mathbf{X}, i=1,2$, be the solutions of equations (1), (2) respectively where $x_{i}(s, s, x)=x$.

Definition 1. The differential equations (1) and (2) are globally dynamical equivalent if there exists a continuous map $H: \mathbb{R} \times \mathbf{X} \rightarrow \mathbf{X}$ such that

(i) for each fixed $t \in \mathbb{R}$ the map $H(t, \cdot): \mathbf{X} \rightarrow \mathbf{X}$ is a homeomorfism;

(ii) $\sup _{t, x}|H(t, x)-x|<+\infty$;

(iii) for all $t \in \mathbb{R}$

$$
H\left(t, x_{1}(t, s, x)\right)=x_{2}(t, s, H(s, x))
$$

Let

$$
D=\left\{(t, s) \in \mathbb{R}^{2} \mid t=s\right\} .
$$

Definition 2. A continuous map $G: \mathbb{R}^{2} \backslash D \rightarrow \mathfrak{L}(\mathbf{X})$ is a Green type map if

(i) $G(\cdot, s)$ is solution of linear differential equation

$$
\dot{x}=A(t) x
$$

except for $t=s$;

(ii)

$$
G(t+0, t)-G(t-0, t)=I
$$

where $I$ is identity map.

Note that the differential equation (3) has infinitely many Green type maps. But if the linear differential equation (3) has an exponential dichotomy then, moreover, there exists an unique Green type map which satisfies the inequality

$$
|G(t, s)| \leq K \exp (-\lambda|t-s|), \quad K \geq 1, \lambda>0 .
$$


Therefore the Green type map can be represented in the form

$$
G(t, s)= \begin{cases}U(t, s) P(s), & \text { if } t>s \\ U(t, s)(P(s)-I), & \text { if } t<s\end{cases}
$$

where $U(t, s)$ is the evolution operator of $(3)$. Let us note that

$$
U(t, \tau) U(\tau, s)=U(t, s)
$$

and the solutions of (1) and (2) can be represented in the form

$$
x_{i}(t, s, x)=U(t, s) x+\int_{s}^{t} U(t, \tau) f_{i}\left(\tau, x_{i}(\tau, s, x)\right) d \tau, \quad i=1,2 .
$$

Theorem 3. Suppose that the linear differential equation (3) has a Green type map $G(s, \tau) \in \mathfrak{L}(\mathbf{X})$ such that

$$
\begin{aligned}
& \sup _{s} \int_{-\infty}^{+\infty}|G(s, \tau)| N(\tau) d \tau<+\infty \\
& \sup _{s} \int_{-\infty}^{+\infty}|G(s, \tau)| \varepsilon(\tau) d \tau=q<1 .
\end{aligned}
$$

Then the systems of differential equations (1) and (2) are globally dynamical equivalent.

Proof. Consider the set of continuous, bounded maps

$$
\mathfrak{M}=\left\{h: \mathbb{R} \times \mathbf{X} \rightarrow \mathbf{X}\left|\sup _{s, x}\right| h(s, x) \mid<+\infty\right\} .
$$

It is easy to see that $\mathfrak{M}$ is a Banach space with the supremum norm

$$
\|h\|=\sup _{s, x}|h(s, x)|
$$

We will seek the map establishing the equivalence of (1) and (2) in the form $H(s, x)=x+h(s, x)$. We examine the following integro-functional equation

$$
\begin{gathered}
h(s, x)=\int_{-\infty}^{+\infty} G(s, \tau)\left(f_{2}\left(\tau, x_{1}(\tau, s, x)+h\left(\tau, x_{1}(\tau, s, x)\right)\right)\right. \\
\left.-f_{1}\left(\tau, x_{1}(\tau, s, x)\right)\right) d \tau .
\end{gathered}
$$


Let us consider the map $h \mapsto \mathfrak{T} h, h \in \mathfrak{M}$ defined by the equality

$$
\begin{gathered}
\mathfrak{T} h(s, x)=\int_{-\infty}^{+\infty} G(s, \tau)\left(f_{2}\left(\tau, x_{1}(\tau, s, x)+h\left(\tau, x_{1}(\tau, s, x)\right)\right)\right. \\
\left.-f_{1}\left(\tau, x_{1}(\tau, s, x)\right)\right) d \tau .
\end{gathered}
$$

Because of boundedness, Lipschitz condition and conditions of the Theorem 3, also $\mathfrak{T} h \in \mathfrak{M}$. Next we get

$$
\begin{gathered}
\left|\mathfrak{T} h(s, x)-\mathfrak{T} h^{\prime}(s, x)\right| \\
=\mid \int_{-\infty}^{+\infty} G(s, \tau)\left(f_{2}\left(\tau, x_{1}(\tau, s, x)+h\left(\tau, x_{1}(\tau, s, x)\right)\right)\right. \\
\left.\quad-f_{2}\left(\tau, x_{1}(\tau, s, x)+h^{\prime}\left(\tau, x_{1}(\tau, s, x)\right)\right)\right) d \tau \mid \\
\leq \int_{-\infty}^{+\infty}|G(s, \tau)| \varepsilon(\tau)\left|h\left(\tau, x_{1}(\tau, s, x)\right)-h^{\prime}\left(\tau, x_{1}(\tau, s, x)\right)\right| d \tau \\
\leq \sup _{s} \int_{-\infty}^{+\infty}|G(s, \tau)| \varepsilon(\tau) d \tau\left\|h-h^{\prime}\right\|=q\left\|h-h^{\prime}\right\|,
\end{gathered}
$$

where $q<1$. Thus the map $\mathfrak{T}$ is a contraction and consequently the integrofunctional equation (4) has a unique solution in $\mathfrak{M}$.

We have

$$
\begin{gathered}
h\left(t, x_{1}(t, s, x)\right)=\int_{-\infty}^{+\infty} G(t, \tau)\left(f_{2}\left(\tau, x_{1}(\tau, s, x)+h\left(\tau, x_{1}(\tau, s, x)\right)\right)\right. \\
\left.-f_{1}\left(\tau, x_{1}(\tau, s, x)\right)\right) d \tau \\
=\int_{-\infty}^{t} G(t, \tau)\left(f_{2}\left(\tau, x_{1}(\tau, s, x)+h\left(\tau, x_{1}(\tau, s, x)\right)\right)\right. \\
\left.-f_{1}\left(\tau, x_{1}(\tau, s, x)\right)\right) d \tau \\
+\int_{t}^{+\infty} G(t, \tau)\left(f_{2}\left(\tau, x_{1}(\tau, s, x)+h\left(\tau, x_{1}(\tau, s, x)\right)\right)\right. \\
\left.-f_{1}\left(\tau, x_{1}(\tau, s, x)\right)\right) d \tau \\
=\int_{-\infty}^{s} U(t, s) G(s, \tau)\left(f_{2}\left(\tau, x_{1}(\tau, s, x)+h\left(\tau, x_{1}(\tau, s, x)\right)\right)\right. \\
\left.-f_{1}\left(\tau, x_{1}(\tau, s, x)\right)\right) d \tau
\end{gathered}
$$




$$
\begin{gathered}
+\int_{s}^{t} U(t, \tau) P(\tau)\left(f_{2}\left(\tau, x_{1}(\tau, s, x)+h\left(\tau, x_{1}(\tau, s, x)\right)\right)\right. \\
\left.-f_{1}\left(\tau, x_{1}(\tau, s, x)\right)\right) d \tau \\
+\int_{s}^{+\infty} U(t, s) G(s, \tau)\left(f_{2}\left(\tau, x_{1}(\tau, s, x)+h\left(\tau, x_{1}(\tau, s, x)\right)\right)\right. \\
\left.-f_{1}\left(\tau, x_{1}(\tau, s, x)\right)\right) d \tau \\
+\int_{t}^{s} U(t, \tau)(P(\tau)-I)\left(f_{2}\left(\tau, x_{1}(\tau, s, x)+h\left(\tau, x_{1}(\tau, s, x)\right)\right)\right. \\
\left.\quad-f_{1}\left(\tau, x_{1}(\tau, s, x)\right)\right) d \tau \\
+\int_{s}^{t} U(t, \tau)\left(f_{2}\left(\tau, x_{1}(\tau, s, x)+h\left(\tau, x_{1}(\tau, s, x)\right)\right)-f_{1}\left(\tau, x_{1}(\tau, s, x)\right) d \tau\right. \\
=U(t, s)\left(f_{2}\left(\tau, x_{1}(\tau, s, x)+h\left(\tau, x_{1}(\tau, s, x)\right)\right)\right. \\
\left.-f_{1}\left(\tau, x_{1}(\tau, s, x)\right)\right) d \tau \\
\int_{s}^{t} U(t, \tau)\left(f_{2}\left(\tau, x_{1}(\tau, s, x)+h\left(\tau, x_{1}(\tau, s, x)\right)\right)\right. \\
\left.-f_{1}\left(\tau, x_{1}(\tau, s, x)\right)\right) d \tau .
\end{gathered}
$$

Consequently, we have

$$
x_{1}(t, s, x)+h\left(t, x_{1}(t, s, x)\right)=x_{2}(t, s, x+h(s, x)) .
$$

Changing the roles of $f_{1}$ and $f_{2}$, we prove in the same way the existence of $h^{\prime}(s, x)$ that satisfies the equality

$$
x_{2}(t, s, x)+h^{\prime}\left(t, x_{2}(t, s, x)\right)=x_{1}\left(t, s, x+h^{\prime}(s, x)\right) .
$$

Designing $H(s, x)=x+h(s, x), H^{\prime}(s, x)=x+h^{\prime}(s, x)$, we get

$$
\begin{aligned}
& H^{\prime}\left(t, H\left(t, x_{1}(t, s, x)\right)\right)=x_{1}\left(t, s, H^{\prime}(s, H(s, x))\right), \\
& H\left(t, H^{\prime}\left(t, x_{2}(t, s, x)\right)\right)=x_{2}\left(t, s, H\left(s, H^{\prime}(s, x)\right)\right) .
\end{aligned}
$$

Taking into account uniqueness of maps $H^{\prime}(t, H(t, \cdot))-I$ and $H\left(t, H^{\prime}(t, \cdot)\right)-$ $I$ in $\mathfrak{M}$ we have $H^{\prime}(t, H(t, \cdot))=I$ and $H\left(t, H^{\prime}(t, \cdot)\right)=I$ and therefore $H(t, \cdot)$ is a homeomorphism establishing a dynamical equivalence of the (1) and (2).

Let $f_{2}(t, x)=0$. Then Theorem 3 implies that differential equations (1) and (3) are globally dynamical equivalent. 


\section{Invertible Difference Equations}

We generalize this result for invertible semilinear difference equations

$$
x(t+1)=A(t) x(t)+f_{1}(t, x(t))
$$

and

$$
x(t+1)=A(t) x(t)+f_{2}(t, x(t))
$$

on $\mathbf{X}$, where the map $A(t) \in \mathfrak{L}(\mathbf{X})$ is invertible, the maps $f_{i}: \mathbb{Z} \times \mathbf{X} \rightarrow \mathbf{X}$, $i=1,2$, satisfy the estimates

$$
\begin{gathered}
\left|f_{1}(t, x)-f_{2}(t, x)\right| \leq N(t)<+\infty \\
\left|f_{i}(t, x)-f_{i}\left(t, x^{\prime}\right)\right| \leq \varepsilon(t)\left|x-x^{\prime}\right| \quad i=1,2
\end{gathered}
$$

and $\sup _{t}\left(\left\|A^{-1}(t)\right\| \varepsilon(t)\right)<1$.

Definition 4. The difference equations (5) and (6) are globally dynamical equivalent if there exists a continuous map $H: \mathbb{Z} \times \mathbf{X} \rightarrow \mathbf{X}$ such that

(i) for each fixed $t \in \mathbb{Z}$ map $H(t, \cdot): \mathbf{X} \rightarrow \mathbf{X}$ is a homeomorfism;

(ii) $\sup _{t, x}|H(t, x)-x|<+\infty$;

(iii) for all $t \in \mathbb{Z}$

$$
H\left(t, x_{1}\left(t, s, x_{s}\right)\right)=x_{2}\left(t, s, H\left(s, x_{s}\right)\right) .
$$

Definition 5. The map $G: \mathbb{Z}^{2} \rightarrow \mathfrak{L}(\mathbf{X})$ is a Green type map if

(i) $G(t+1, s)=A(t) G(t, s), \quad t \neq s-1$;

(ii) $G(t, t)=A(t) G(t-1, t)+I$.

Theorem 6. Suppose that the linear difference equation

$$
x(t+1)=A(t) x(t)
$$

has a Green type map $G(t, s) \in \mathfrak{L}(\mathbf{X})$ such that

$$
\begin{aligned}
& \sup _{t \in \mathbb{Z}} \sum_{s=-\infty}^{+\infty}|G(t, s+1)| N(s)<+\infty, \\
& \sup _{t \in \mathbb{Z}} \sum_{s=-\infty}^{+\infty}|G(t, s+1)| \varepsilon(s)=q<1
\end{aligned}
$$

then the difference equations (5) and (6) are globally dynamical equivalent. 
Proof. To prove Theorem 6, we prove that the following functional equation

$$
\begin{gathered}
h\left(s, x_{s}\right)=\sum_{i=-\infty}^{+\infty} G(s, i+1)\left(f_{2}\left(i, x_{1}\left(i, s, x_{s}\right)+h\left(i, x_{1}\left(i, s, x_{s}\right)\right)\right)\right. \\
\left.-f_{1}\left(i, x_{1}\left(i, s, x_{s}\right)\right)\right)
\end{gathered}
$$

has unique continuous and bounded solution. The proof is similar to the proof for differential equations.

\section{Example}

Consider the system of quasilinear differential equations in $\mathbb{R}^{3}$

$$
\dot{x}=A(t) x+f(t, x)
$$

where

$$
\begin{gathered}
A(t)=\left(\begin{array}{ccc}
0 & -1 & 0 \\
1 & 0 & 0 \\
0 & 0 & -\frac{2 t}{1+t^{2}}
\end{array}\right) \\
\left|f(t, x)-f\left(t, x^{\prime}\right)\right| \leq \varepsilon(t)\left|x-x^{\prime}\right|
\end{gathered}
$$

and

$$
\sup _{x}|f(t, x)| \leq N(t)<+\infty .
$$

Then the fundamental matrix of the system linear differential equations takes the form

$$
U(t, s)=\left(\begin{array}{ccc}
\cos (t-s) & -\sin (t-s) & 0 \\
\sin (t-s) & \cos (t-s) & 0 \\
0 & 0 & \frac{1+s^{2}}{1+t^{2}}
\end{array}\right)^{.}
$$

The corresponding Green type map can be represented in the form

$$
\begin{gathered}
G(t, s)=\left(\begin{array}{ccc}
0 & 0 & 0 \\
0 & 0 & 0 \\
0 & 0 & \frac{1+s^{2}}{1+t^{2}}
\end{array}\right), \text { if } t>s \\
G(t, s)=\left(\begin{array}{ccc}
\cos (t-s) & -\sin (t-s) & 0 \\
\sin (t-s) & \cos (t-s) & 0 \\
0 & 0 & 0
\end{array}\right), \text { if } t<s .
\end{gathered}
$$


If

$$
\sup _{t} \int_{-\infty}^{+\infty}|G(t, s)| \varepsilon(s) d s \leq \int_{-\infty}^{+\infty}\left(1+s^{2}\right) \varepsilon(s) d s<1
$$

and

$$
\sup _{t} \int_{-\infty}^{+\infty}|G(t, s)| N(s) d s \leq \int_{-\infty}^{+\infty}\left(1+s^{2}\right) N(s) d s<+\infty
$$

then, in accordance with Theorem 3, the system (8) is globally dynamically equivalent to the linear one

$$
\dot{x}=A(t) x .
$$

The system (9) does not even have an ordinary dichotomy [5].

\section{Acknowledgements}

This work was partially supported by the grant Nr. 345/2012 of the Latvian Council of Science.

\section{References}

[1] D.M. Grobman, Topological classification of neighbourhoods of a singularity in $n$-space, Mat. Sb., 56, No. 1 (1962), 77-94 (in Russian).

[2] P. Hartman, On the local linearization of differential equations, Proc. Amer. Math. Soc., 14, No. 4 (1963), 568-573, doi: 10.1090/S0002-99391963-0152718-3.

[3] C.C. Pugh, On a theorem of P. Hartman, Amer. J. Math., 91, No. 2 (1969), 363-367, doi: 10.2307/2373513.

[4] K.J. Palmer, A generalization of Hartman's linearization theorem, Math. Anal. Appl., 41, No. 3 (1973), 752-758, doi: 10.1016/0022-247X(73)90245$\mathrm{X}$.

[5] W.A. Coppel, Dichotomies in Stability Theory, Springer-Verlag, Berlin Heidelberg New York (1978).

[6] Liangping Jiang, Generalized exponential dichotomy and global linearization, Math. Anal. Appl., 315, No. 2 (2006), 474-490, doi: 10.1016/j.jmaa.2005.05.042. 
[7] Liangping Jiang, Ordinary dichotomy and global linearization, Nonlinear Anal., Theory Methods Appl., 70, No. 7 (2009), 2722-2730, doi: 10.1016/j.na.2008.03.060.

[8] Xiaodan Chen and Yonghui Xia, Topological conjugacy between two kinds of nonlinear differential equations via generalized exponential dichotomy, Int. J. Differ. Equ., (2011), doi: 10.1155/2011/871574.

[9] Yonghui Xia, Xiaodan Chen and V. Romanovski, On the linearization theorem of Fenner and Pinto, J. Math. Anal. Appl., 400, No. 2 (2013), 439-451, doi: 10.1016/j.jmaa.2012.11.034.

[10] Yongfei Gao, Xiaoqing Yuan, Yonghui Xia and P.J.Y. Wong, Linearization of impulsive differential equations with ordinary dichotomy, Abstr. Appl. Anal., (2014), doi: 10.1155/2014/632109.

[11] Yongfei Gao, Yonghui Xia, Xiaoqing Yuan and P.J.Y. Wong, Linearization of nonautonomous impulsive systems with nonuniform exponential dichotomy, Abstr. Appl. Anal., (2014), doi: 10.1155/2014/860378.

[12] A. Reinfelds, On generalized Grobman-Hartman theorem, Latv. Mat. Ezhegodnik, 29 (1985), 84-88 (in Russian).

[13] A. Reinfelds, Grobman's-Hartman's theorem for time-dependent difference equations, Latv. Univ. Zinātn. Raksti, 605 (1997), 9-13.

[14] A. Reinfelds, Asymptotic equivalence of difference equations in Banach space, in: Theory and Applications of Difference Equations and Discrete Dynamical Systems, Springer Proc. Math. Statist., 102 (2014), pp. 215222, doi: 10.1007/978-3-662-44140-4_ 12 . 